\title{
Introduction
}

\author{
Looking at Labor
}

We may capture the portrait of history in so-called insignificant visual and verbal textualities and textiles. In material details. In twill fabrics, bead-work pieces, pricked patterns, four-ringed knots, tiny spangles, sharptoothed stencil wheels; in quotations, thought-fragments, rhymes, syllables, anagrams, graphemes, endangered phonemes, in soils and cross-outs.

- SUSAN HOWE ${ }^{1}$

\section{FRAGMENT BY FRAGMENT}

Imagine studying a building not by walking its hallways or perusing its blueprints, but by examining each of its bricks: the pockmarks produced by air bubbles in the clay, the whorls of reds and browns, the trowel's impressions in the mortar. Imagine evaluating a mosaic not for the bigger picture but for the glint of individual tesserae. Or imagine not watching a film but looking at it frame by frame. Bodies in motion would suddenly freeze, their irresistible sensuousness submitting to clinical scrutiny. Minute details in the photographic image would supplant the broader strokes of the narrative. The part would overwhelm the whole.

But each of those fragments affords its own pleasures and hints at its own story. In this book, I use the fragments to piece together a portrait of cinema history and theory. I focus in particular on US animated cartoons, a tremendous body of work long excluded from the study of film proper. The animated cartoons I examine were produced with the technique of cel animation, which gets its name from the transparent sheets of cellulose nitrate or acetate ("cels") on which cartoon characters were painted. Cel animation is now a moribund medium, kept alive only by independent practitioners, but it was the dominant technique for most of the twentieth century. Its basic parts were interchangeable, which facilitated high-volume production and made it particularly amenable to standardization and mechanization. From the 1920s through the 1960s, the classical era of US animation, major studios like Walt Disney, Leon Schlesinger / Warner Bros., Fleischer, Walter Lantz, 
MGM, and United Productions of America used the cel animation technique to produce hundreds of seven-minute films each year. Animation was an industry as much as it was an artistic medium. By arresting the animation of animation, I aim to return cartoons to how they were made: one drawing at a time, one photograph at a time, one frame at a time. Through this mode of very close analysis, I provide an account of the aesthetics of an art formed on the assembly line.

In order for Mickey Mouse (or Bugs Bunny or Popeye or Woody Woodpecker or Tom and Jerry) to move on-screen, thousands of cels had to be photographed in succession, a highly labor-intensive process that was divided across a factory of artists and technicians. One group of animators was responsible for the initial sketches, another for painting these drawings onto cels, a third for taking each photograph. But the paradox of cartoons is that all of this carefully choreographed work disappears the moment the image springs into motion. The knowledge that Mickey Mouse is nothing more than ink and paint cannot overcome the perception that he is alive. By viewing the film frame by frame, however, one can spot the traces of the hundreds of hands that touched Mickey before he made his way to the screen. Sometimes these assume the form of stray brushstrokes or strands of hair, and sometimes they linger as oily smudges, the literal fingerprints of the workers who handled the image. Such traces reveal aspects of the animation process that the viewer was never intended to see, but their discovery does not undermine the film's aesthetic power. Instead, the cartoon assumes new historical weight: even if the world it conjures up is populated by anthropomorphic animals and governed by impossible physics, it becomes apparent that its constitutive elements were real, material things: pen and paper and glass and celluloid.

The photographic process is thus not simply an incidental step in the industrial production of animated cartoons. Camera technology invests animated cartoons with the same evidentiary force as any work of photography. It says, This object existed, this object was made by human hands. Studied frame by frame, photograph by photograph, animated cartoons serve a powerful documentary function. They show us parts of our world. At the same time, they can be placed into larger theoretical debates about the nature of technological reproduction as such, for instance the relationship between image and text, the fraught authorship of popular art, and the political implications of the circulation of hitherto inaccessible works of art.

This book thus takes seriously the photographic image's dual status-as a document that testifies to the existence of a specific time and place, and as a work of art with its own affective power. Its governing tension lies between the knowledge one can obtain through photographic evidence and the aesthetics of those photographs. What can images tell us, and how do they speak? A photograph of a cel might reproduce a coagulated stroke of ink, but I can only guess at the cause of its coagulation. There is a limit to what the photograph alone can show. Yet the pursuit of that limit itself constitutes a form of aesthetic experience. This mode of looking is the very object of my study. 


\section{FRAGMENTATION}

First devised by Earl Hurd and John Randolph Bray in the mid-1910s, the cel animation technique was predicated on the principles of scientific management. As advanced by Frederick Taylor and Frank Gilbreth, scientific management broke the labor process down to discrete movements, allowing for its measurement and rationalization. ${ }^{2}$ The earliest patents for cel animation proposed a method of dividing labor between "the artist," who provided an original sketch, and "an assistant," who did not need to possess "the originality, skill, or ability of an artist" in order to perform his or her primary tasks of tracing and painting. An influential how-to manual first published in 1920 picked up the language of the patents in its recommendation of "a division of labor between the animator and his helper," whereby "the animator does the first planning and that part of the subsequent work requiring true artistic ability," while "the actual toil of repeating monotonous details falls upon the tracer." ${ }^{\text {"3 }}$ Dividing the tasks of animating (performed by a skilled artist) from those of tracing (performed by an unskilled laborer) and other steps in the production process enabled studios to speed up production, reduce labor costs, and standardize their product.

While the division prescribed by the patents was not unique to cel animation (for instance, a rudimentary division of labor was enacted between the pioneering animator Winsor McCay and his assistant, John Fitzsimmons), the process's technological components, particularly the cels themselves, paved the way for the implementation of assembly-line production. There was no conveyor belt per se. Rather, the introduction of interchangeable parts married the Taylorist emphasis on efficiency and standardization to the Fordist model of mechanization of the labor process. Moreover, by eliminating the sorts of technical errors to which rival techniques like McCay's artisanal model or Raoul Barrés slash-and-tear system were prone, the process and the product alike were streamlined. ${ }^{4}$

There was variation in how tasks broke down from studio to studio, and some (for instance Disney) had higher degrees of rationalization and regulation than others (for example Fleischer and Warner Bros.). 5 This book focuses on the work of four departments that no studio went without: the Animation Department, the Ink Department, the Paint Department, and the Camera Department. The first of these comprised several positions, including that of the head animator, who supervised the animation of a particular scene or character. His job was to oversee the drawing of the "extremes," the most dynamic poses in a given action, and he often had assistant animators to aid him in the task. His crew also included in-betweeners, who were responsible for all the intermediary poses that came between one extreme and the next, and clean-up artists, who erased the stray lines from the rough sketches. The men who held the latter positions were generally considered noncreative or "below-the-line" laborers, but they had the potential for promotion to creative or "above-the-line" positions as 
assistant or head animators. The drawings the Animation Department churned out-up to twenty-four per second, or one for every frame of film, but usually no more than sixteen-would then be sent to the Ink and Paint Departments for transfer onto cels. The inkers (who traced the drawings onto cels) and the painters (who colored inside the inked lines) were almost exclusively women. Although they had to undergo significant training, their work was noncreative, and they had no expectation of promotion to above-the-line positions. Finally, the inked and painted cels would make their way to the Camera Department to be photographed by below-the-line technicians. Detailed exposure sheets provided by the cartoon's director instructed the camera operator on the number and relative placement of the cels to be photographed, the positions of both the camera and the painted background relative to the cels, and the number of times each of these cel setups (as many as four cels atop a background) was to be photographed.

All of these tasks were repetitive and demanding, performed for long hours and little pay. In the 1930s, unionization efforts were undertaken at two of the studios then based in New York-an unsuccessful drive at Van Beuren, followed by a successful strike at Fleischer Studios in 1937. In 1941 the Los Angeles studios encountered their first wave of labor unrest, the most significant being the strike by workers at Walt Disney. This book, while not a traditional industrial or labor history, takes these early unionization efforts as the original inspiration for its questions about the relationship between art and labor. ${ }^{6}$ I rely on primary sources, particularly interviews and oral histories, in order to understand better how animators (as well as inkers, painters, and camera operators) conceived of that relationship. Importantly, while all the early strikes in the animation industry concentrated on standard labor grievances, they were also anchored in a romantic notion of artistic autonomy. But I do not consider their answers definitive. Instead, they underscore the difficulty of disentangling the creative from the noncreative, intention from accident or inevitability, work as such from the work of art. Ultimately, my interest lies in how this knowledge-of technology and of laborbears (or should bear) on the viewing of the animated cartoons themselves. During the strike at Fleischer Studios, union members picketed theaters and on occasion would interrupt screenings with Bronx cheers and cries of "Take that scab picture off the screen!"7 Eighty years hence, I am not willing to boycott Popeye cartoons. Even so, I want to watch them in a way that recognizes their invested labor and technology as integral to their artistry.

Does knowing about a particular technical process shape what we are able to see-indeed, how we see? Conversely, can we learn to treat these films as sites of potential knowledge - that is, as primary evidence, equal in their documentary value to patents, interviews, trade press articles, and technical manuals? In pursuit of answers, I have studied 1,625 animated cartoons released between 1915 and 1965, 
a period that begins with the first cel animation patent and ends with the industry's shift away from shorts intended for theatrical exhibition, toward commercials and programs for television. ${ }^{8}$ These films ranged from installments in beloved series like Disney's Silly Symphonies and Warner Bros.' Merrie Melodies and Looney Tunes to the output of lesser-known studios like Terrytoons and Screen Gems. Many, if not most, of them amount to little more than corny ephemera. They are repetitive: plots, gags, and individual animation sequences reappear from film to film. (How many dachshunds were tied into knots in the 1920 s and 1930s? How many sticks of dynamite were set ablaze in the 1940s and 1950s?) Yet even the most slapdash among them manages to evince what Vivian Sobchack has called the "seductive spontaneity" characteristic of the medium-a spontaneity so seductive, in fact, that it belies the highly regulated production apparatus that makes it possible. ${ }^{9}$ Here is where our knowledge of the labor process comes into conflict with our aesthetic experience of the films themselves. It seems one cannot be integrated into the other.

A common solution to this problem is to let the films themselves perform the critiques. There are countless examples, starting with the very advent of the form, of self-reflexive, quasi-Brechtian animated cartoons that purport to show us how they were made. In the pre-cel era, these included the vaudevillian films of McCay and J. Stuart Blackton, who starred as magicians of the pen endowed with the power of cinema to conjure living drawings before our eyes. As Donald Crafton has shown, the trope of the animator's self-figuration continued through the early 1920s, with the early cartoons of Hurd, Fleischer, and Lantz. But soon the magic of the animators was transferred to their creations, which were granted the ability to animate themselves; thus Felix the Cat and Oswald the Lucky Rabbit and Mickey Mouse could miraculously improvise anything-propellers, punctuation marks, staircases-out of their own bodies. ${ }^{10}$ The putative hand of the artist would continue to resurface, most famously in Disney's The Three Caballeros (1944) and Chuck Jones's Duck Amuck (Warner Bros., 1953), but these hands were always animated themselves.

Building on Crafton's observations, scholars have since identified other ways in which the production process was represented on-screen. Kirsten Moana Thompson, for example, has argued that the labor of the inkers and painters was brought to the fore in the spectacular Technicolor displays of 1930 os Disney films, and Peng-yi Tai's dissertation on the animation industry, which opens with the assertion that "animation is a record of a labor process," analyzes how assembly-line production is represented in the films of Disney and Fleischer. Nicholas Sammond's recent book on the intertwined histories of animation and blackface minstrelsy makes a compelling case that the fluid figures of Felix et al. were displacements of the animators' longing for rebellion. ${ }^{11} \mathrm{Scott}$ Bukatman, too, has examined the rebellion of such characters as Little Nemo 
and Pinocchio, in the course of which he provides a tidy formulation of one of the chief concerns of this book:

Writers on animation continually circle around the tension between the anarchic polymorphic perversity that it presents and the hypperregulated mode of production that produces it. Animation as an idea speaks to life, autonomy, movement, freedom, while animation as a mode of production speaks to division of labor, precision of control, abundances of preplanning, the preclusion of the random. ${ }^{12}$

What follows, while indebted to these analyses, departs from them in a significant way. Only one chapter, the fourth, considers the narrative content of a film, Disney's One Hundred and One Dalmatians (1961), at any length. What I offer might be considered formal analysis, insofar as I provide an account of the visual style born of specific technologies and techniques, but I do not argue that the aesthetic properties I am interested in have any expressive function-at least not by design. My focus is on the incidental and the accidental, the qualities of the image that resist being understood as the product of creative intention: the textures of a graphic mark, the patterns of paint splatter, jarring collages, swirling specks of dust. The random, as it turns out, is not necessarily precluded from the production of animation. "How do you maintain a sense of improvisation in a cartoon that is built out of thousands of drawings and dozens of tests?" Scott Curtis asks. ${ }^{13}$ To answer him, I show that there resides a spontaneity as seductive as the metamorphoses of cartoon bodies in those parts of the image that rebel against the coherence of the whole. And by attending to those details, those fragments, I revivify the divided labor process.

\section{ANYTHING CAN HAPPEN. . .}

This is not how cartoons have ever been watched. They are meant to be seen in motion, as objects of pure pleasure. They grant not knowledge but instead, crucially, what Sergei Eisenstein called "obliviousness." And this is precisely their appeal: they give us access to limitless worlds in which the impossible is possible. Writing in the early 1940s, Eisenstein praised Walt Disney for granting viewers a momentary respite from "the suffering caused by the social conditions of the social order of the largest capitalist government." ${ }^{14}$ Walter Benjamin, in an essay published in 1933, posited that Mickey Mouse held out "a dream for contemporary man." ${ }_{15}$ The escape animated cartoons offered might have been fleeting or illusory, but it was nonetheless urgent. "We cartoon characters can have a wonderful life, if we only take advantage of it," Jeckle tells Heckle in The Power of Thought (Eddie Donnelly / Terrytoons, 1948). "We can do anything we think of." "Anything can happen in a cartoon," writes Richard Thompson in 1971, paraphrasing Tex Avery's Big Heel-Watha (MGM, 1944): "In a cartoon, you can do anything." In 2014 Bukatman continues the refrain, drawing this time on Friz Freleng's Peck 
Up Your Troubles (Warner Bros., 1945): "Anything is possible in a cartoon."16 The fundamental appeal of animated cartoons, whether they star Elmer Fudd or Gerald McBoing-Boing, Gandy Goose or Screwy Squirrel, lies in the entrancing mutability of their worlds.

Siegfried Kracauer once praised Max Fleischer's Ko-Ko the Clown for his "captivating silliness." ${ }^{17}$ But three decades later, in his influential Theory of Film (1960), he would shunt animation aside on the grounds that "the best method of getting at [film's] core is to disregard, at least temporarily, its least essential ingredients and varieties." ${ }^{18}$ Indeed, the obvious artificiality of cartoons (as the historian Eric Smoodin enumerates, "the animals that spoke and danced, the ease with which objects changed shape or color, the painted rather than realistic mise-en-scène") arguably contributed to their long exclusion from film theory and history. ${ }^{19}$ First published more than thirty years ago, Kristin Thompson's "Implications of the Cel Animation Technique" (1980) remains a compelling account of the medium's marginalization, first within Hollywood and later within the domain of academic film studies. Thompson centers her attention on the formal and technical features that distinguish cel animation from live action:

The crucial aspect of cel animation is its separation of the different foreground and background layers. Typically, the background layer(s) remain constant throughout a shot, while the cels for the moving figures must be frequently redrawn. This difference in the amount of work involved [in] the background and foreground tends to promote a split between the types of depth cues used in the separate layers. . . . In practice, this visual difference between backgrounds and figures has led to a considerable mixing of whole perspective systems within single films. ${ }^{20}$

These formal properties, when fully exploited, threatened the stable codes of live-action narrative feature filmmaking. In order to assimilate such "potentially disruptive" characteristics into the Hollywood system, animation had to be recast as mere children's entertainment. Live-action films could thereby in part be defined-and their primacy reinforced-by how they differed from the trivial cartoon: "Since disruption unmotivated by narrative is unwelcome in the classical system, Hollywood needed to tame the technology," Thompson writes. "Trivialization provided the means." ${ }^{21}$ While the historical dimension of her argument may be somewhat post hoc, ergo propter hoc in its narrative, her larger observations are nonetheless refreshing. She takes this marginalized form seriously, centering the bulk of her analysis on the relationship between film technology and film style in order to provide a rigorous account of why animated cartoons look the way they do.

The 1980 s and 1990s saw the publication of several important popular and academic works on the industrial and cultural history of animation. ${ }^{22}$ Neglected, however, was any sustained engagement with the theoretical and aesthetic issues posed by cel animation as both a technology and a technique. ${ }^{23}$ It is only in recent 
years that scholars have begun to pick up where Thompson left off. Indeed, much has changed in the last decade alone. In addition to some of the works cited above, numerous articles and books now offer vital insights into the formal properties of cel animation. These include Daniel Goldmark's and Lea Jacobs's studies of cartoon soundtracks; Thomas Lamarre's and Casey Riffel's examinations of the spatial qualities of the flat layers of cel setups; Tom Klein's and Scott Curtis's auteurist studies of, respectively, Shamus Culhane and Tex Avery; and Donald Crafton's and Dan Bashara's histories of cartoon style in, respectively, the 1930s and the 1950s. ${ }^{24}$ Jacobs, who draws on bar and exposure sheets-tools used by animators to track and record information about music and cels over the time of the cartoon-in her research on sound-image relations in early Disney cartoons, quotes William Garity, a technician at the studio:

The director and the musician work hand in hand, measure by measure, frame by frame; each one trying to adjust his particular problem to meet the demands of the story. When the layout sheet is completed, the director has this picture completely laid out to the frame, and the musician his master note to the score. Slight changes may later be made in order to accommodate the exigencies that may arise when the pictures are animated. ${ }^{25}$

Measure by measure, frame by frame, note by note, Jacobs then reconstructs the production process that made possible the silliest of symphonies, treating the films as primary sources as rich with historical significance as Garity's remarks. Klein, meanwhile, uses a frame-by-frame study of the animated cartoons of Shamus Culhane to expose the abstract "mini-films" that lurk within seemingly innocuous Woody Woodpecker cartoons, thereby unsettling the history of cartoon poetics. Their attention to detail is matched by that of the other scholars I have listed. With the obsolescence of the cel animation technique has come the rebirth of serious criticism of animated cartoons.

We can attribute some of this shift, too, to the rise of new media technologies and digital cinema, which have reshaped the landscape of film studies more broadly. By throwing into flux many established presuppositions about the ontology of the photographic image, new media have made space for the consideration of forms and formal aspects of cinema that theories such as Kracauer's had necessarily bracketed. Lev Manovich's The Language of New Media, published at the turn of the twenty-first century, has become the pivotal source for identifying one implication in particular:

Manual constructions and animation of images gave birth to cinema and slipped into the margins ... only to reappear as the foundation of digital cinema. The history of the moving image thus makes a full circle. Born from animation, cinema pushed animation to its periphery, only in the end to become one particular case of animation. ${ }^{26}$ 
Through the heuristic of digital cinema, all of cinema can now be recast and reappraised as animation. The margins haven't come to the center-they've become the frame. While Manovich does not alter how we look at animated cartoons per se, he at least opens up that possibility.

Tom Gunning, in turn, provides an important revision of film theory that aims in part to integrate a consideration of animation. For Gunning, animation's exclusion from film theory is one of the discipline's "great scandals," an unintended consequence of the "photographic understanding of cinema." ${ }^{27}$ By revealing the ontological instability of photography, new media allow us to direct our attention to another property specific to the filmic medium, one that has been taken for granted for too long: the movement of moving images, the motion of motion pictures, the kinesis of cinema-and the animation of animation. In a follow-up essay, Gunning builds on this claim by arguing that animation triggers wonder in "its pivot from stillness to motion," a transformation that "reveals the single frame, the brief incremental of time, through the possibility of motion." 28

But can the still frame alone inspire wonder? Can the individual frame offer escapism, oblivion, impossibility? My desire to arrest movement, to view animated cartoons not in motion but as a series of stills, tacitly acknowledges the primal power of movement to occlude the labor behind it. To view an animated cartoon in pieces, one motionless image after another, affords a precision of vision that allows us to see the strokes that constitute cartoon characters, which stand as physical traces of the lowest craft worker. One travels through the photograph, through the camera lens, through the glass platen that holds the cels in place, toward the individual layers of cels, each of which has been inked and painted by hand-and from these cels back toward the original animation drawings from which they were traced. The single frame is a palimpsest, a document, a fragment. But what happens to art? And what happens to aesthetic experience?

\section{THE MONTAGE OF FRAGMENTS}

Laura Mulvey has described the meaning and the beauty that can be found in "some detail or previously unnoticed moment," the discovery of which is made possible through a form of "interactive spectatorship"; Christian Keathley has suggested that these kinds of details "are the sites of both a challenge to historiographic practice and an opportunity for its transformation"; and Garrett Stewart has claimed that the single frame's "function in the apparatus of cinema . . . is so widely overlooked in commentary that to reclaim its somewhat demonstrable (and always necessary) contribution counts as a theoretical proposal in its own right." ${ }^{29}$ These three arguments - all closely related, but the first primarily aesthetic, the second historiographical, and the third theoretical-intertwine in my approach to the study of animated cartoons. Looking at cartoons frame by frame enables me to think of each frame as the photograph it is, which brings them into conversation 
with the discourses of photography in film theory from which they have been excluded. Moreover, each frame is a documentary record of a moment in time, which gives it new historical significance. And the very process of going through a film $1 / 24$ th of a second at a time, cross-referencing frames between films, creating catalogues of recycled images and abstract images, and uncovering otherwise obscured details-all operations I perform in the course of this book-becomes an act of play.

I am aided in my mode of analysis by digital technology, which has made films more accessible and, as a consequence, easier to manipulate. Of course, prior to the arrival of home-video formats, scholars were able to conduct close analysis using film prints, and many dwelled on individual shots or even frames. ${ }^{30}$ The difference is a matter of degree-but so large is the degree that it might very well be one of kind, too. Without such resources as DVDs and YouTube, not to mention QuickTime, Final Cut Pro, and Adobe Photoshop, I would not have been able to watch the hundreds of shorts that I did, nor amass such a vast archive of images with just a few keystrokes.

Ultimately, the way I watch cartoons is the way Stan Brakhage watched On Duty (1973), the colorized version of One More Time (Rudolf Ising / Warner Bros., 1931), and one of the sources for his found-footage film Murder Psalm (1980); it is the way Standish Lawder watched Paul Terry's Fox Hunt (1927), the source for two of his studies in rephotography and optical printing, Runaway (1970) and Roadfilm (1970); it is the way both Bruce Conner and Martin Arnold watched classic Mickey Mouse cartoons, which Conner quotes in Cosmic Ray (1962) and Arnold dissects in Soft Palate and Shadow Cuts (both 2010). And it is the way Robert Breer, an experimental animator whose films I cite in each chapter, watched the work of Émile Cohl and Otto Messmer, to whom he pays tribute in LMNO (1978) and Rubber Cement (1976). As Robert Ray has argued vis-à-vis "Cubist collages, Surrealist film-watching habits, Duchamp's readymades, Pop Art's cartoons and soup cans, [and] Joseph Cornell's flea-market boxes," the fetishistic attention to detail and repurposing of found fragments that characterize these avant-garde films are, in fact, "methods of research." ${ }^{31}$ And, in turn, my methodology is a form of montage.

It is thus noteworthy that several seminal shot-by-shot studies have been devoted to the films of Sergei Eisenstein..$^{32} \mathrm{He}$ is, along with Walter Benjamin, one of the thinkers central to this book, not only for his influential writings on Walt Disney-which Scott Bukatman has deemed "the ur-text of animation studies" but also for his theory and practice of montage. ${ }^{33}$ Anne Nesbet has called him and Benjamin surrealist philosophers, a term Theodor Adorno used derisively but which she finds, at the very least, provocative. Both Eisenstein and Benjamin, she notes, reveled in finding junctures "between seemingly disparate objects or epochs." ${ }^{34}$ Their theoretical practice, in other words, had consequences for the writing of history-and they delighted in it as if it were an aesthetic experience. 
Indeed, Ivor Montagu remembers that Eisenstein "taught us to see much with new eyes." 35 I train this new vision on animated cartoons.

\section{CHAPTER OUTLINE}

The poet Susan Howe, an admirer of both Eisenstein and Benjamin, has noted that writers like Herman Melville and Emily Dickinson were all "using montage before it was a word for a working method.” Curiously, Jay Leyda, one of Eisenstein's protégés, used montage to write documentary biographies of both Melville and Dickinson, the first of which he dedicated to his mentor. Howe's description of Melville's and Dickinson's method applies to Leyda's-as well as Benjamin's and Eisenstein's: “Their writing practice (varied though it was) involved comparing and linking fragments or shots, selecting fragments for scenes, reducing multitudes (chapters or stanzas) and shots (lines and single words) to correlate with one another, constantly interweaving traces of the past to overcome restrictions of temporal framing." ${ }^{36}$ The first chapter of this book likewise seeks to resist what I call the sequential logic of animation. Through an examination of alternative organizational models such as Eisenstein's imagined "spherical book," Benjamin's "mosaic," Leyda's biographies, Dickinson's poetry, and Robert Breer's Blazes (1961), I posit that animation, too, can be reconfigured as montage. In fact, many animated cartoons contain moments that serve to disrupt the linearity of the filmstrip, breaking the forward flow of the animation. These include flicker sequences that consist of the rapid alternation of distinct frames, which causes the animation to stutter and strobe, and expository inserts such as newspapers, which grind the animation to a halt. By overcoming the filmstrip's linear logic, these sequences invite us to treat them as if they were fragments of a montage-comparing them, linking them, interweaving them.

The first chapter forms a diptych with the second, which argues that animated cartoons can be understood as photographic records of their own production. Both chapters emphasize the form's connection to photographic practices and discourses - in the first, to cinematic montage and single-frame cinematography, and, in the second, to realist and structuralist/materialist theories of cinematic ontology. A model for this method is the work of the artist Andrew Norman Wilson, whose ScanOps (2012) consists of a series of photographs culled from Google Books. Just as Wilson reveals that the scanner and the scanner's operator are not incidental to Google's digitization practices, I aim to show how the camera and the camera operator played an active role in creating animated cartoons. Therefore, taking as its basic premise the fact that all works of cel animation were photographic in origin, chapter 2 demonstrates the ways in which the physical reality of our world, and particularly the world of the animation studio, leaves its mark on the cartoon image. I catalogue the various visual imperfections that testify to cel animation's photographic origins. These include improperly placed cels, reflections of the 
camera apparatus, dust and dirt particles, and even fingerprints left by anonymous laborers. Although these mistakes may only appear on the screen for a fraction of a second, each has been preserved for posterity as a still photograph.

The problem of the "mistake" is then complicated in the third chapter, which marks a shift away from photographic accounts of animation, toward its graphic characteristics. I look in particular at cels painted by anonymous female laborers whose work it is that we ultimately see on-screen. This chapter aims to challenge the division between creative and noncreative labor by imagining that these paintings might contain "deliberate mistakes," an oxymoron that exposes the impossibility of knowing artistic intent in works of mass art. Although our knowledge here encounters an absolute limit, we can nonetheless speculate about what such mistakes might look like: the frame-by-frame examination of numerous cartoons uncovers countless instances in which representation falls apart, as when famous cartoon characters are distorted beyond all recognition (usually in order to simulate graphically a photographic effect, such as motion blur). I then pose a counterfactual: What if every frame contained these mistakes? Once again, this might seem like an unsolvable riddle, but, once again, the films themselves raise a possible answer: the continuous unspooling of the filmstrip - the sequential logic the first chapter resists-readily assimilates even the most discontinuous of images.

It is only in the final chapter that I offer a means of bringing my mode of close analysis to bear on formal analysis. Looking at cartoons frame by frame illuminates the relationship between form and content, the medium and the message, the means of production and the narrative. Furthermore, chapter 4 comes closest to a traditional history of film style and technology, charting how changes in the US animation industry of the 1950 altered the aesthetics of popular cartoons. As I show, rising production costs and competition from television forced many studios to downsize or even send work overseas. Walt Disney, for one, eliminated an entire branch of his studio-the Inking Department. The method of manual reproduction that had held sway for four decades was supplanted by xerographic reproduction, also known as photocopying. Disney's One Hundred and One Dalmatians (1961), the central case study, is a film that could not have been made without the Xerox machine, as well as a film about the Xerox machine. A close analysis of its visual style reveals how cel animation is shaped both by the demands of mass production and by technologies of reproduction.

Each of these chapters thus provides a way of looking at the art, labor, and technology of cel animation. The first treats each frame as the photographic reproduction of a historical document, the second as a photograph proper, the third as a graphic image, the fourth as text. These are thought experiments. To look in each of these ways in turn and in conjunction requires concentration. A cubist hermeneutic, it strains the eye and tests the limits of plausibility, with a view toward the impossible. In animated cartoons, anything can happen. 